\title{
Do seismic waves sense fracture connectivity?
}

\author{
J. Germán Rubino, ${ }^{1}$ Luis Guarracino, ${ }^{2}$ Tobias M. Müller, ${ }^{3}$ and Klaus Holliger ${ }^{1}$ \\ Received 16 November 2012; revised 21 December 2012; accepted 24 December 2012.
}

[1] A defining characteristic of fractured rocks is their very high level of seismic attenuation, which so far has been assumed to be mainly due to wave-induced fluid flow (WIFF) between the fractures and the pore space of the embedding matrix. Using oscillatory compressibility simulations based on the quasi-static poroelastic equations, we show that another important, and as of yet undocumented, manifestation of WIFF is at play in the presence of fracture connectivity. This additional energy loss is predominantly due to fluid flow within the connected fractures and is sensitive to their lengths, permeabilities, and intersection angles. Correspondingly, it contains key information on the governing hydraulic properties of fractured rock masses and hence should be accounted for whenever realistic seismic models of such media are needed. Citation: Rubino, J. G., L. Guarracino, T. M. Müller, and K. Holliger (2013), Do seismic waves sense fracture connectivity?, Geophys. Res. Lett., 40, doi:10.1002/grl.50127.

\section{Introduction}

[2] Fractures are common features in virtually all geological formations and tend to dominate not only their mechanical, but also, and especially, their hydraulic properties. For these reasons, the detection and characterization of fractures are of great interest in several domains in the Earth, environmental, and engineering sciences, such as groundwater and contaminant hydrology, nuclear waste storage, $\mathrm{CO}_{2}$ sequestration, hydrocarbon exploration and production, and tunnel engineering, among many others [e.g., Liu et al. 2000; Nelson, 2001; Maultzsch et al., 2003].

[3] The analysis of fractures through direct observations, such as outcrop or borehole studies, is problematic due to the inherently limited sampling of the affected rock volumes. Hence, remote-sensing approaches are of significant interest. Geophysical techniques in general and seismic methods in particular are therefore valuable for the non-invasive detection and characterization of fractures [e.g., Maultzsch et al., 2003; Clark et al., 2009]. Since the resolving power of typical seismic data is generally not high enough to directly image fractures, most research efforts for fracture characterization

\footnotetext{
${ }^{1}$ Applied and Environmental Geophysics Group, University of Lausanne, Lausanne, Switzerland.

${ }^{2}$ CONICET, Facultad de Ciencias Astronómicas y Geofísicas, Universidad Nacional de La Plata, Buenos Aires, Argentina.

${ }^{3}$ Earth Science and Resource Engineering Division, Commonwealth Scientific and Industrial Research Organization, Perth, Australia.

Corresponding author: J. G. Rubino, Applied and Environmental Geophysics Group, University of Lausanne, CH-1015 Lausanne, Switzerland. (German.Rubino@unil.ch)

(C) 2013. American Geophysical Union. All Rights Reserved. $0094-8276 / 13 /$ grl.50127
}

focus on attribute analysis [e.g., Bakulin et al., 2000]. Notably, seismic attenuation has been recognized as a critical attribute for this purpose, as both laboratory studies and field measurements provide evidence that seismic energy loss is very significant in fractured rocks and tends to increase with increasing fracture density [e.g., Peacock et al., 1994; Maultzsch et al., 2003; Clark et al., 2009].

[4] Up until now, the high seismic attenuation observed in fractured rocks has been considered to be predominantly produced by wave-induced fluid flow (WIFF) between fractures and the pore space of the embedding matrix [e.g., Peacock et al., 1994; Chapman, 2003; Brajanovski et al., 2005; Gurevich et al., 2009]. Since the wave-induced fluid pressure equilibration process is strongly influenced by the hydraulic characteristics of the fractured rock, one should expect that the resulting seismic attenuation also contains information about fracture connectivity. Fracture connectivity controls the flow and transport properties of fractured formations [e.g., Berkowitz et al., 2000; Renshaw, 1996] and hence, relevant information extracted from seismic observations would be of great importance. To date, however, the effects of fracture connectivity with regard to the observed seismic attenuation are largely unexplored, which is possibly due to the inherent limitations of commonly employed effective medium models [e.g., Hudson et al., 1996; Chapman, 2003; Brajanovski et al., 2005].

[5] In this paper, we seek to explore the effects of fracture connectivity on seismic attenuation through the use of a numerical upscaling procedure. This approach enables us to estimate $P$ wave attenuation due to WIFF in complex fractured media and thus allows us to relate fracture connectivity effects to seismic attenuation with unprecedented realism.

\section{Methodological Background}

[6] The seismic response of fractured porous rocks can be modeled in the framework of Biot [1962] theory of poroelasticity by representing the fractures as highly compliant and permeable heterogeneities embedded in a stiffer porous matrix [Brajanovski et al., 2005; Gurevich et al., 2009]. The large compressibility contrasts between the background material and the fractures hence produce a significant oscillatory flow from the fractures into the host rock and vice versa in response to the propagation of seismic waves, thus causing energy dissipation. The spatial scales at which this oscillating flow occurs is characterized by the diffusion length $L_{\mathrm{d}}^{\mathrm{b}}=\sqrt{D^{\mathrm{b}} / \omega}$, where $\omega$ is the angular frequency of the seismic wave and $D^{\mathrm{b}}$ is the pressure diffusivity of the background material, a physical parameter that is directly proportional to the permeability $\kappa^{\mathrm{b}}$. Maximum attenuation occurs at the characteristic frequency $\omega_{\mathrm{c}}^{\mathrm{b}}$ for which the diffusion length $L_{\mathrm{d}}^{\mathrm{b}}$ and the characteristic length scale of the heterogeneities $a_{h}$ are similar. Therefore, $\omega_{\mathrm{c}}^{\mathrm{b}} \approx D^{\mathrm{b}} / a_{h}^{2}$. 
[7] We employ a numerical upscaling procedure similar to that presented by Rubino et al. [2009] to estimate $P$ wave attenuation due to WIFF. In this sense, we apply an oscillatory compression at the top boundary of a two-dimensional rectangular poroelastic medium containing mesoscale fractures, that is, fractures larger than the pore size but smaller than the prevailing wavelengths. No tangential forces are applied on the boundaries of this synthetic sample and the solid is allowed neither to move on the bottom boundary nor to have horizontal displacements on the lateral boundaries. The fluid is not allowed to flow into or out of the sample. The equivalent undrained, complex plane-wave modulus is expressed in terms of the time-harmonic compression and the complex oscillatory volume change. The latter is obtained by numerically solving the quasi-static poroelastic equations in the space-frequency domain under the governing boundary conditions. This complex, frequency-dependent modulus yields the attenuation and phase velocity corresponding to the vertically propagating $P$ wave.

[8] To analyze fracture connectivity effects on $P$ wave attenuation caused by WIFF, we consider a poroelastic model of a homogeneous water-saturated sandstone permeated by a set of mesoscale fractures. The background material has a solid bulk modulus $K_{\mathrm{s}}^{\mathrm{b}}=37 \mathrm{GPa}$, a shear modulus $\mu_{\mathrm{s}}^{\mathrm{b}}=44$ $\mathrm{GPa}$, a density $\rho_{\mathrm{s}}^{\mathrm{b}}=2.65 \mathrm{~g} / \mathrm{cm}^{3}$, and a porosity $\phi^{\mathrm{b}}=0.1$. To compute the drained-frame parameters, Krief's relations and the formula of Kozeny-Carman [e.g., Mavko et al., 2009] are employed to account for the relationships between the elastic moduli, the permeability, and the porosity. This yields the drained-frame bulk and shear moduli $K_{\mathrm{m}}^{\mathrm{b}}=23 \mathrm{GPa}$ and $\mu_{\mathrm{m}}^{\mathrm{b}}=27 \mathrm{GPa}$, and a permeability $\kappa^{\mathrm{b}}=23 \mathrm{mD}$. We assume that the solid material properties of the fractures are the same as those of the background. Following Nakagawa and Schoenberg [2007], we characterize the elastic properties of the drained fracture in terms of the shear compliance $\eta_{T}=h / \mu_{\mathrm{m}}^{\mathrm{f}}$ and drained normal compliance $\eta_{N}=h /\left(K_{\mathrm{m}}^{\mathrm{f}}+\frac{4}{3} \mu_{\mathrm{m}}^{\mathrm{f}}\right)$, where $h$ is the fracture thickness and $K_{\mathrm{m}}^{\mathrm{f}}$ and $\mu_{\mathrm{m}}^{\mathrm{f}}$ are the drained-frame bulk and shear moduli of the fracture.

[9] Figure 1 shows the three synthetic rock samples used to analyze the sensitivity of $P$ wave attenuation to fracture connectivity. The two-dimensional samples are squares of side length $2.5 \mathrm{~cm}$ and the mean thickness of the fractures is $h=0.033 \mathrm{~cm}$. Using the values $\eta_{T}=3 \times 10^{-11} \mathrm{~m} / \mathrm{Pa}$ and $\eta_{N}=10^{-11} \mathrm{~m} /$ Pa proposed by Nakagawa and Schoenberg [2007], we obtain $K_{\mathrm{m}}^{\mathrm{f}}=0.02 \mathrm{GPa}$ and $\mu_{\mathrm{m}}^{\mathrm{f}}=0.01 \mathrm{GPa}$. Also, we assume a fracture permeability of $\kappa^{\mathrm{f}}=100 \mathrm{D}$ and a porosity $\phi^{\mathrm{f}}=0.5$. Fractures and background are fully saturated with water with bulk modulus $K_{\mathrm{w}}=2.25 \mathrm{GPa}$, density $\rho_{\mathrm{w}}=1.09 \mathrm{~g} / \mathrm{cm}^{3}$ and shear viscosity $\eta_{\mathrm{w}}=0.003 \mathrm{~Pa} \mathrm{~s}$.

\section{Results}

[10] The bottom panel of Figure 1 shows the computed inverse quality factor $Q_{p}^{-1}$ for vertically incident $P$ waves as a function of frequency for the three samples shown in the top panels of the same figure. The red curve indicates
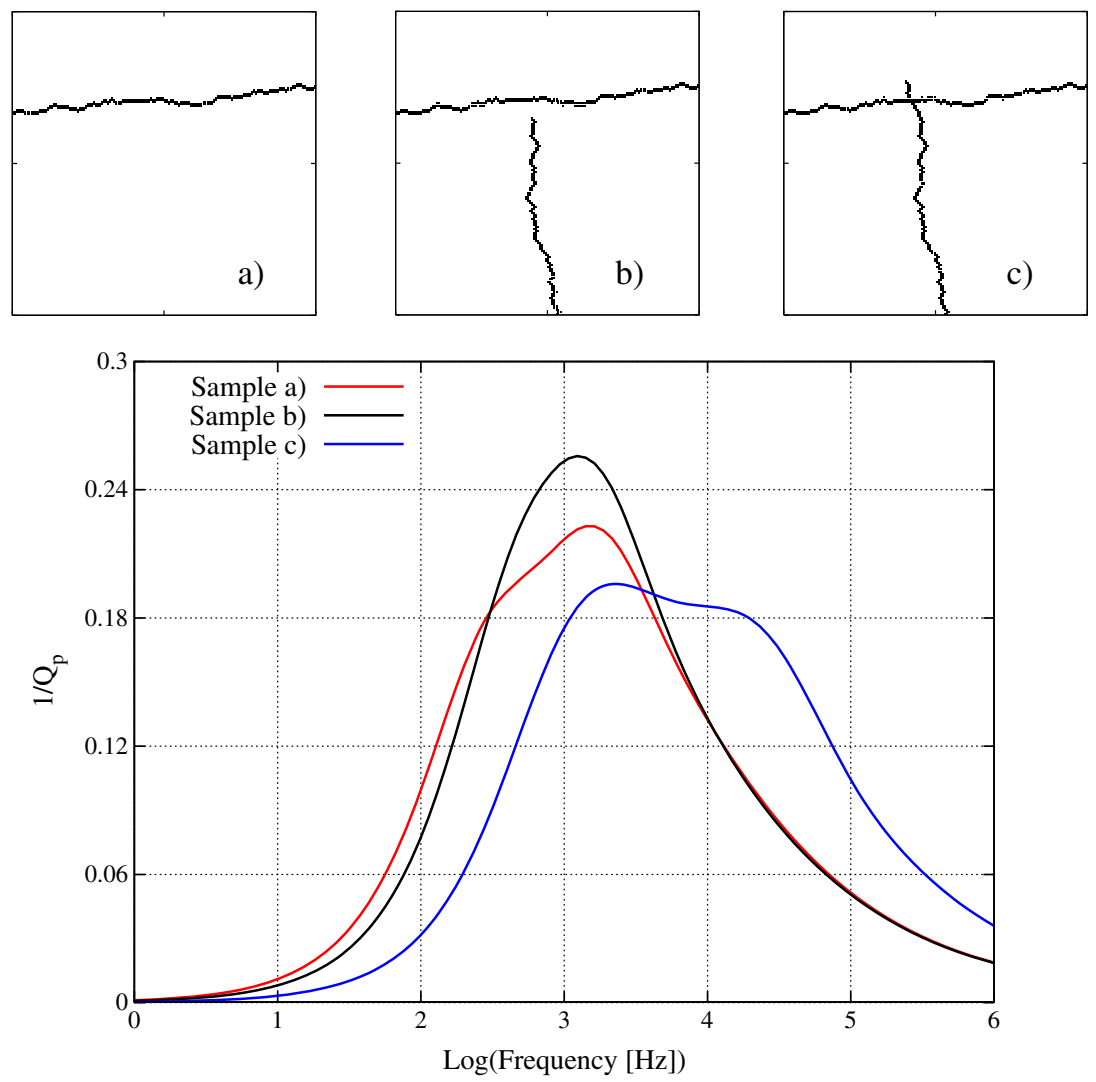

Figure 1. Synthetic rock samples (top panels) employed to analyze fracture connectivity effects on $P$ wave attenuation. Fractures are denoted by solid "wiggly" lines. Sample a) contains a single sub-horizontal fracture, while samples b) and c) contain two quasi-orthogonal fractures without and with intersection, respectively. The bottom panel shows the corresponding inverse $P$ wave quality factor as a function of frequency for a vertical direction of propagation. 
that the presence of fractures perpendicular to the direction of wave propagation produces very strong attenuation, with $Q_{p}$ values below 20 over a wide frequency range. By comparing the black and red curves we see that adding a non-intersecting fracture parallel to the direction of wave propagation only has a moderate effect with regard to the maximum attenuation. This is expected, since the vertical compression applied to the sample does not change significantly the fluid pressure in the vertical fracture and, consequently, there is no significant WIFF. However, as the vertical fracture intersects the horizontal one, significant changes in the overall attenuation behavior arise (blue curve). We observe that the maximum attenuation decreases slightly and that the frequency range where attenuation is relevant broadens. Moreover, there seem to be two local maxima at $\sim 1.3$ and $\sim 29 \mathrm{kHz}$. The first attenuation peak occurs at a frequency similar to the transition frequency of the red and black curves, thus indicating that it may be due to fluid exchange between the fractures and the background material. Conversely, the presence of a second peak at higher frequencies may be indicative of an additional energy loss caused by the intersection of the two fractures.

[11] In order to corroborate the latter hypothesis, we show in Figure 2 the real part of the fluid pressure field normalized with respect to the amplitude of the stress applied on the top boundary of the sample, computed at the frequencies of the local maxima, that is, $1.3 \mathrm{kHz}$ (left panels) and $29 \mathrm{kHz}$ (right panels). The top and bottom panels correspond to samples b) and c) in Figure 1, respectively.

[12] The top left panel of Figure 2 shows that the applied compression produces a significant fluid pressure increase in the highly compliant, horizontal fracture as compared to the stiffer background rock, thus producing significant fluid flow across the boundary of fracture and background rock. This WIFF results in attenuation as depicted by the black curve in the bottom panel in Figure 1. Note that the fluid pressure induced within the vertical fracture for sample b) is relatively low so that the presence of this fracture does not change significantly the overall fluid pressure field.

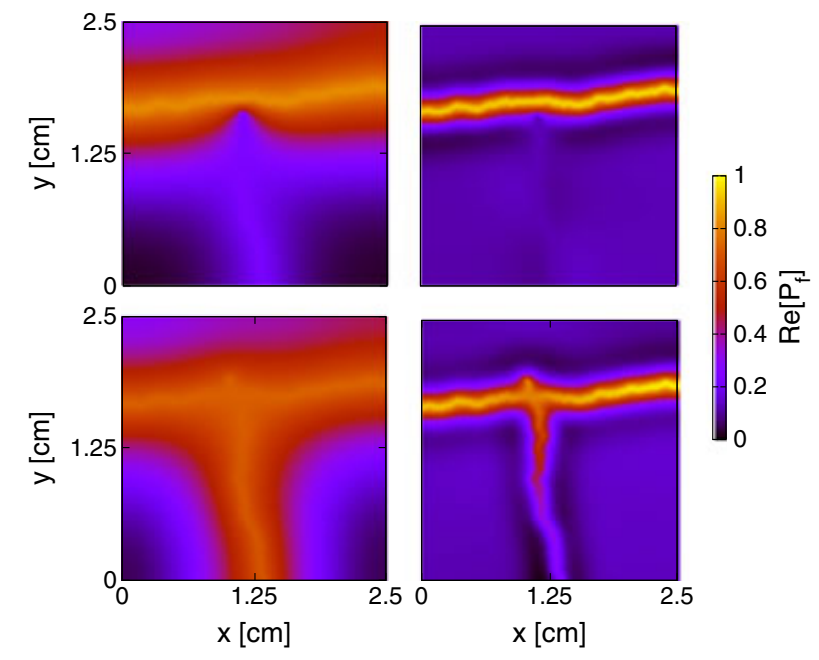

Figure 2. Real part of the fluid pressure normalized with respect to the amplitude of the stress applied on the top boundaries of the synthetic rock samples, for frequencies of $1.3 \mathrm{kHz}$ (left panels) and $29 \mathrm{kHz}$ (right panels). Top and bottom panels correspond to the rock samples b) and c) in Figure 1, respectively.
However, when the vertical fracture intersects the horizontal one (sample c), the fluid pressure field changes rather substantially, as can be seen in the bottom left panel of Figure 2. We observe that the fluid pressure in the vertical fracture increases significantly while the horizontal fracture experiences a slight fluid pressure reduction as compared to the unconnected scenario (top left panel). This altered pressure distribution is due to the hydraulic connectivity between the two fractures and, correspondingly, the amount of fluid flowing between the horizontal fracture and the background rock decreases. However, fluid flow between the fractures and background rock also takes place in the vicinity of the vertical fracture, albeit at a less significant level. The bottom panel in Figure 1 illustrates that these changes in the WIFF pattern have corresponding effects on the attenuation behavior.

[13] For the frequency at which the second attenuation peak occurs, fluid pressure changes in the background matrix are limited to the immediate vicinity of the fractures (right panels of Figure 2). This is expected because the corresponding diffusion lengths become smaller as frequency increases. Accordingly, fluid exchange between the horizontal fracture and the background rock is much less significant for this higher frequency. Again, the presence of the vertical fracture does not change significantly the fluid pressure field when there is no connection. However, when the fractures intersect, the fluid pressure field changes substantially, as illustrated by the comparison of the top and bottom right panels. Interestingly, for this higher frequency there is a significant fluid pressure gradient and corresponding fluid flow inside the vertical fracture. Fluid is injected from the horizontal fracture which experiences an increase of fluid pressure in response to the vertical compression. Hence, while fluid flow within the horizontal fracture is not very pronounced when there is no fracture intersection, it becomes substantial when there is fracture intersection, as we verified by analyzing the horizontal component of the real part of the relative fluid-solid displacement.

[14] These considerations clearly indicate that at the frequency of the second attenuation peak WIFF is much more significant in the presence of connected fractures and occurs predominantly within the fractures, thus making this phenomenon the most likely explanation of the corresponding additional attenuation peak. To further corroborate this hypothesis, we decrease the permeability of the background material from $\kappa^{\mathrm{b}}=23 \mathrm{mD}$ to $\kappa^{\mathrm{b}}=0.023 \mathrm{mD}$ and recompute the resulting attenuation. Attenuation peaks associated with fluid flow in the background should then shift toward lower frequencies since the corresponding transition frequency $\omega_{\mathrm{c}}^{\mathrm{b}}$ is directly proportional to $\kappa^{\mathrm{b}}$, whereas those resulting from flow within fractures should remain unchanged. The results shown in Figure 3 confirm that when there is no fracture connection (blue curves) the attenuation peak moves toward lower frequencies when decreasing the background rock permeability. For connected fractures (red curves), the peak originally located at a frequency of $1.3 \mathrm{kHz}$ also shifts toward lower frequencies (dashed red line), whereas the attenuation peak at $29 \mathrm{kHz}$ remains at the same location. These results clearly demonstrate that the second attenuation peak is not a result of fluid flow between the fractures and the embedding matrix, but rather related to fluid flow within the fractures. In the following, we analyze some key features of this new manifestation of WIFF. 


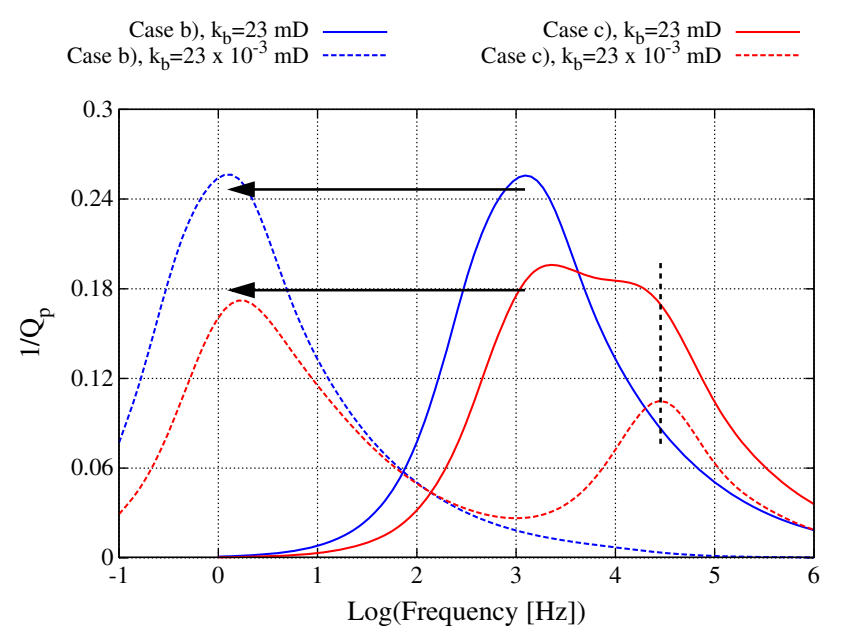

Figure 3. Inverse $P$ wave quality factor as a function of frequency. Blue and red colors correspond to the rock samples b) and c) in Figure 1, respectively. Solid line patterns indicate that the original permeability value is used for the background material $\left(\kappa^{\mathrm{b}}=23 \mathrm{mD}\right)$, while dashed patterns correspond to the responses obtained using a significantly lower permeability $\left(\kappa^{\mathrm{b}}=0.023 \mathrm{mD}\right)$.

\section{Characteristics of Attenuation Due to Flow Within Fractures}

[15] If the permeability of the embedding matrix is much smaller than that of the fracture, it can be neglected from a hydraulic point of view. Consequently, the location of the attenuation peak related to flow within fractures is determined by the frequency at which the diffusion length in the fracture material is of similar size as the fracture length. That is, the corresponding transition frequency is given by $\omega_{\mathrm{c}}^{\mathrm{f}} \approx D^{\mathrm{f}} / a_{\mathrm{f}}^{2}$, where $D^{\mathrm{f}}$ is the pressure diffusivity of the fracture material and $a_{\mathrm{f}}$ is the fracture length. Conversely, if the background permeability is comparable to that of the fracture material, the fluid will have enough time during each half-cycle to flow from the fracture into the background and vice versa and, hence, the above relation between $\omega_{\mathrm{c}}^{\mathrm{f}}$ and $a_{\mathrm{f}}$ does not hold any longer.

[16] For the particular material properties and geometries employed in the simulations, we verified that for connected fractures the transition frequency of the first attenuation peak $\omega_{\mathrm{c}}^{\mathrm{b}}$ is not sensitive to the fracture permeability, while the second attenuation peak shifts toward higher frequencies with increasing $\kappa^{\mathrm{f}}$ for values above $\sim 1 \mathrm{D}$. However, for $\kappa^{\mathrm{f}}$ values below $\sim 1 \mathrm{D}$, the attenuation curves are not very sensitive to the fracture permeability, and the aforementioned relation between $\omega_{\mathrm{c}}^{\mathrm{f}}$ and $\kappa^{\mathrm{f}}$ is not valid. We also confirmed that $\omega_{\mathrm{c}}^{\mathrm{f}}$ depends on the lengths of the fractures.

[17] As can be seen in Figure 3, the energy loss associated with flow within the fractures occurs at frequencies well beyond the seismic band. However, the relationship between $\omega_{\mathrm{c}}^{\mathrm{f}}$ and the fracture properties indicates that for other fracture geometries or physical parameters this frequency range can shift toward lower or higher values. Hence, the findings of this study can be expected to scale throughout the seismic, sonic, and ultrasonic frequency bands. In addition, if the second attenuation peak is located outside the seismic frequency band, fracture connectivity may also affect the behavior of the first attenuation peak, as shown in Figure 1. This means that even in such unfavorable cases seismic attenuation may encode some information on fracture connectivity.

[18] We also analyzed the role of the intersection angle by considering two fractures of equal length intersecting at their centers. One of the fractures is located horizontally in the center of the rock sample and intersected by the other one at an angle $\theta$. We observed that the intersection angle does not change significantly the location of the two attenuation peaks but results in significant magnitude changes. In particular, we found that the second peak is highest for $\theta=90^{\circ}$ and is reduced as $\theta$ decreases. Conversely, the magnitude of the first peak is lowest for $\theta=90^{\circ}$ and increases as $\theta$ decreases.

[19] Finally, it is important to mention that this new manifestation of WIFF arising in the presence of fracture connectivity can be regarded as the mesoscopic analogue of the microscopic squirt flow mechanism proposed by Mavko and Nur [1975]. This microscopic attenuation mechanism requires the hydraulic interconnection of two or more grain-scale cracks of different orientation and, hence, the application of stress causes fluid flow from one microscopic crack into another, thus producing seismic energy dissipation.

\section{Conclusions}

[20] We have found that the presence of mesoscale fractures oriented quasi-perpendicular to the direction of wave propagation produces very significant seismic attenuation due to WIFF between fractures and the embedding porous matrix. Conversely, the addition of fractures quasi-parallel to the propagation direction does not produce significant changes to the attenuation curves, provided that the fractures do not intersect. However, as soon as the fractures intersect, the attenuation behavior changes significantly. We observed a slight decrease in the magnitude of the attenuation peak corresponding to the unconnected situation together with the occurrence of an additional prominent attenuation peak in the presence of fracture connectivity. Our results demonstrate that this additional and as of yet unknown manifestation of WIFF is produced by flow within the connected fractures. Since all fractured media have a certain degree of interconnectivity, this additional WIFF contribution must be accounted for in order to realistically model seismic attenuation in such environments.

[21] Given that the attenuation produced by this newly identified WIFF mechanism contains information not only about fracture connectivity, but also about the permeability as well as the length and intersection angle of the fractures, a deeper understanding of this process will reveal interesting perspectives for the seismic monitoring and characterization of fractured rocks.

[22] Acknowledgments. This work was supported in part by grants from the Swiss National Science Foundation, the Herbette Foundation of the University of Lausanne, and the Agencia Nacional de Promoción Científica y Tecnológica (PICT 2010-2129), Argentina. The authors thank Ranajit Ghose and an anonymous reviewer, as well as the editor Michael Wysession for lucid and constructive comments that helped to improve the quality of this manuscript.

\section{References}

Bakulin, A., V. Grechka, and I. Tsvankin (2000), Estimation of fracture parameters from reflection seismic data-Part I: HTI model due to a single fracture set, Geophysics, 65, 1788-1802.

Berkowitz, B., O. Bour, P. Davy, and N. Odling (2000), Scaling of fracture connectivity in geological formations, Geophys. Res. Lett., 27, 2061-2064. 


\section{RUBINO ET AL.: SEISMIC WAVES AND FRACTURE CONNECTIVITY}

Biot, M. (1962), Mechanics of deformation and acoustic propagation in porous media, J. Appl. Phys., 33, 1482-1498.

Brajanovski, M., B. Gurevich, and M. Schoenberg (2005), A model for Pwave attenuation and dispersion in a porous medium permeated by aligned fractures, Geophys. J. Int., 163, 372-384.

Chapman, M. (2003), Frequency-dependent anisotropy due to meso-scale fractures in the presence of equant porosity, Geophys. Prospect., 51, 369-379.

Clark, R., P. Benson, A. Carter, and C. Guerrero Moreno (2009), Anisotropic P-wave attenuation measured from a multi-azimuth surface seismic reflection survey, Geophys. Prospect., 57, 835-845.

Gurevich, B., M. Brajanovski, R. Galvin, T. Müller, and J. Toms-Stewart (2009), P-wave dispersion and attenuation in fractured and porous reservoirs-Poroelasticity approach, Geophys. Prospect., 57, 225-237.

Hudson, J., E. Liu, and S. Crampin (1996), The mechanical properties of materials with interconnected cracks and pores, Geophys. J. Int., 124, 105-112.

Liu, E., J. Hudson, and T. Pointer (2000), Equivalent medium representation of fractured rock, J. Geophys. Res., 105, 2981-3000.

Maultzsch, S., M. Chapman, E. Liu, and X. Li (2003), Modelling frequencydependent seismic anisotropy in fluid-saturated rock with aligned fractures:
Implication of fracture size estimation from anisotropic measurements, Geophys. Prospect., 51, 381-392.

Mavko, G., and A. Nur (1975), Melt squirt in the asthenosphere, $J$. Geophys. Res., 80, 1444-1448.

Mavko, G., T. Mukerji, and J. Dvorkin (2009), The Rock Physics Handbook: Tools for Seismic Analysis of Porous Media, 2nd ed., Cambridge Univ. Press, New York, 524 pp.

Nakagawa, S., and M. Schoenberg (2007), Poroelastic modeling of seismic boundary conditions across a fracture, J. Acoust. Soc. America, 122, 831-847.

Nelson, R. (2001), Geologic Analysis of Naturally Fractured Reservoirs, 2nd ed., Gulf Professional Publishing, Oxford, UK

Peacock, S., C. McCann, J. Sothcott, and T. Astin (1994), Experimental measurements of seismic attenuation in microfractured sedimentary rock, Geophysics, 59, 1342-1351.

Renshaw, C. (1996), Influence of subcritical fracture growth on the connectivity of fracture networks, Water Resour. Res., 32, 1519-1530.

Rubino, J., C. Ravazzoli, and J. Santos (2009), Equivalent viscoelastic solids for heterogeneous fluid-saturated porous rocks, Geophysics, 74, N1-N13. 